\title{
Eosinophilic Esophagitis in Children: Updates in Diagnosis and Management
}

\section{Minou Le-Carlson ${ }^{1}$ and J. Antonio Quiros ${ }^{2 *}$}

${ }^{1}$ Fellow, Division of Gastroenterology, Lucille Packard Children's Hospital, Stanford University School of Medicine, Stanford, CA, USA

${ }^{2}$ Chief, Department of Pediatric Gastroenterology and Nutrition. Associate Clinical Professor of Pediatrics, Pediatric Center for Inflammatory Bowel Disorders, California

Pacific Medical Center, San Francisco, CA, USA

\section{Introduction}

Eosinophilic esophagitis (EoE) is a chronic, allergen and immune mediated condition that is characterized by the abnormal infiltration of eosinophils in the esophageal mucosa. Part of the family of eosinophilic gastrointestinal diseases, EoE has emerged as a distinct disease process with unique clinical and pathophysiologic characteristics. EoE has garnered much attention within the medical community as demonstrated by an explosion of research since its first description in the literature in the 1970's. Also increasing are the incidence (1.5 per 10,000) and prevalence (5 per 10,000) of EoE due to heightened awareness and an overall rise in number of cases. Untreated EoE can have significant effects on children including growth changes, chronic pain and irreversible fibrosis. The goal of the pediatric gastroenterologist is to identify and diagnosis EoE. Early diagnosis of disease can lead to effective treatment interventions, prevention of complications and improved quality of life for patients. Here, we explore the diagnosis and management of EoE in the children from the perspective of the pediatric gastroenterology specialist.

\section{Diagnosis}

As discussed in the $2011 \mathrm{EoE}$ consensus report, EoE is based on both clinical and histologic findings. To meet histologic criteria, there must be 15 or more eosinophils per high power field (HPF) in 1 or more biopsies (Figure 1). Patients should demonstrate upper gastrointestinal symptoms typical for esophageal dysfunction such as epigastric pain. Other causes of eosinophilic inflammation such as gastro-esophageal reflux disease (GERD) and infection must be excluded. A trial of acid suppressive therapy should be undertaken for at least 6 weeks prior to any diagnostic maneuver to effectively rule out GERD. Lastly, clinical and histopathologic improvement should develop following interventions such as diet modifications and/or medications [1]. Based on this definition, eosinophilic inflammation that occurs with other diseases such as celiac disease or inflammatory bowel disease does not meet criteria for EoE but instead should be labeled a more general form of esophageal eosinophilia.

Symptom presentation in children tends to vary by age. Infants and toddlers often present with feeding difficulty and irritability. School-age children typically have signs of emesis and chest pain, while teenagers experience symptoms of dysphagia $[2,3]$. The challenge for the pediatric gastroenterologist is identifying whvich of these patients presenting with such nonspecific symptoms should be further evaluated for EoE with endoscopy. Studies have shown increase rates of EoE in certain populations that may be helpful guiding the clinician. For instance, $40-90 \%$ of EoE patients have a history of atopy such as eczema, food allergies, asthma and/or allergic rhinitis. Additionally, there is a clear predominance in males, $>3: 1$, for reasons that are not known [1]. The conventional approach is treat patients with upper gastrointestinal symptoms suggestive of GERD with acid supression using a proton pump inhibitor (PPI) like omeprazole at $1 \mathrm{mg} / \mathrm{kg} /$ dose twice daily, for a minimum of 2 months, and if symptoms persist despite adequate antacid therapy then to proceed with endoscopy. Diagnosis can be complicated by the subset of EoE patients who demonstrate clinical improvement with PPI therapy, referred to as PPI-responsive esophageal eosinophilia. Partial improvement with PPI may be a contributing factor as to why diagnosis is often delayed-an average of 3 years between onset of symptoms and diagnostic endoscopy in one study [4]. At this time this issue should not dissuade physicians caring for children with suspected EoE from following the current guidelines.

\section{Endoscopy}

Endoscopy performed by a trained pediatric gastroenterologist is needed to diagnose EoE. While under anesthesia, a flexible endoscope is advanced, through the mouth into the patient's esophagus, stomach and duodenum. In these areas, the mucosa is visualized to identify any gross evidence of pathology and small mucosal biopsies are taken Studies have shown the diagnostic sensitivity to be $84 \%$ with 2 biopsies, but increases to $97 \%$ and $100 \%$ with 3 and 6 respectively which is likely due to the patchy pattern of inflammation [5]. Sampling both proximal and distal levels aids in distinguishing between EoE and GERD. Eosinophilic infiltration in the proximal esophagus is rarely noted in GERD while it is very typical in EoE. Approximately $10 \%$ of EoE patients demonstrate no obvious macroscopic changes. Conversely, of the patients who exhibit gross features suggestive of disease, less than $40 \%$ actually have histologic evidence of EoE. Given the above, multiple biopsies from more than one level should be obtained during endoscopy, even in the setting of grossly normal appearing tissue [1].

Normal mucosa appears smooth with no irregularities (Figure 1C). In EoE, there a range of nonspecific changes in the esophagus in that can be observed. Typical features include mucosa that appears erythematous, dull and thickened. White plaques or eosinophilic abscesses can be present and with more chronic inflammation, linear furrows or circumferential rings (Figure 1A)[2]. Luminal narrowing and strictures, fortunately are rare findings in children. Extra care should be used during endoscopy to prevent traumatizing inflamed tissue particularly in young children; multiple cases of perforation with endoscopy biopsy and dilatation have occurred [6]. Resistance during

*Corresponding author: J. Antonio Quiros, MD, FAAP, 3700 California Street Suite 1560, San Francisco, CA 94118, USA, Tel: (415)600-0743; Fax: (415)6000741; E-mail: quirosj@sutterhealth.org

Received October 25, 2011; Accepted December 17, 2011; Published December 22, 2011

Citation: Minou Le-Carlson, J Antonio Quiros (2011) Eosinophilic Esophagitis in Children: Updates in Diagnosis and Management. J Aller Ther S8:001. doi:10.4172/2155-6121.S8-001

Copyright: (C) 2011 Minou Le-Carlson, et al. This is an open-access article distributed under the terms of the Creative Commons Attribution License, which permits unrestricted use, distribution, and reproduction in any medium, provided the original author and source are credited. 
Citation: Minou Le-Carlson, Antonio Quiros J (2011) Eosinophilic Esophagitis in Children: Updates in Diagnosis and Management. J Aller Ther S8:001. doi:10.4172/2155-6121.S8-001

the procedure may suggest stiff, noncompliant tissue or narrowing and thus warrants the use of a smaller endoscope. Dilatation procedures for stricture formation in children have been shown to be safe and effective with experienced gastroenterologists.

As studies have shown, the degree of esophageal inflammation does not directly correlate to patient symptoms. It is also not clear how often repeat endoscopies should be performed to monitor the disease. At this time, no clear guidelines have been outlined. The consensus committee recommends the goal of treatment should be the resolution of inflammation to prevent longterm sequelae such as irreversible fibrosis and stricture formation [1]. EoE is a chronic condition, and tends be progressive if untreated. Thankfully only a small percentage of cases evolve into stricture formation. At this time, it is not possible to identify who is most at risk of developing major complications of their EoE. Indeed, research to investigate whether surveillance endoscopy actually improves outcomes in the long-term care of patients is highly needed. Also under investigation is whether certain serologic markers such as eosinophil-derived neurotoxin can accurately correlate with esophageal inflammation, and be used in lieu of endoscopy to stage disease [7].

Endoscopic ultrasound (EUS) has also been found to be a useful tool in EoE management. EUS involves the examination of the esophageal wall by using safe, high-frequency ultrasound, delivered by a through-

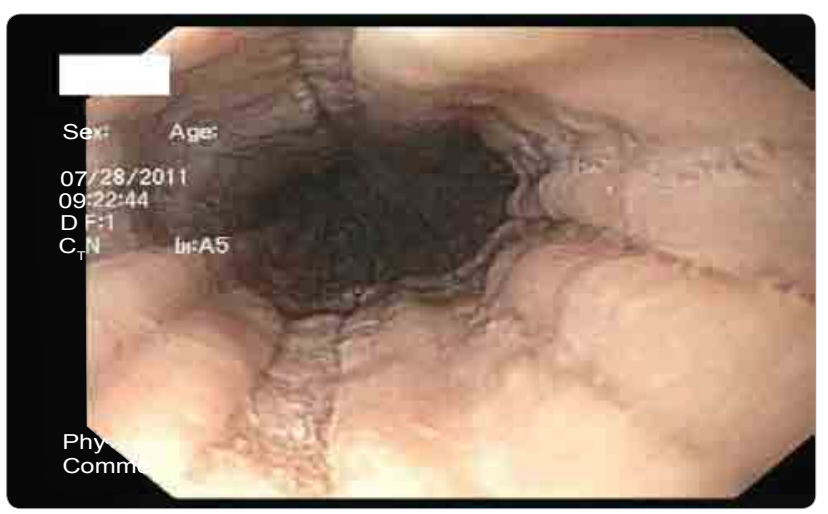

Figure 1a: 15 yo caucasian male with a history of atopy, seasonal allergies and new onset dysphagia. On initial endoscopy focal exudates and linear furrowing was noted.

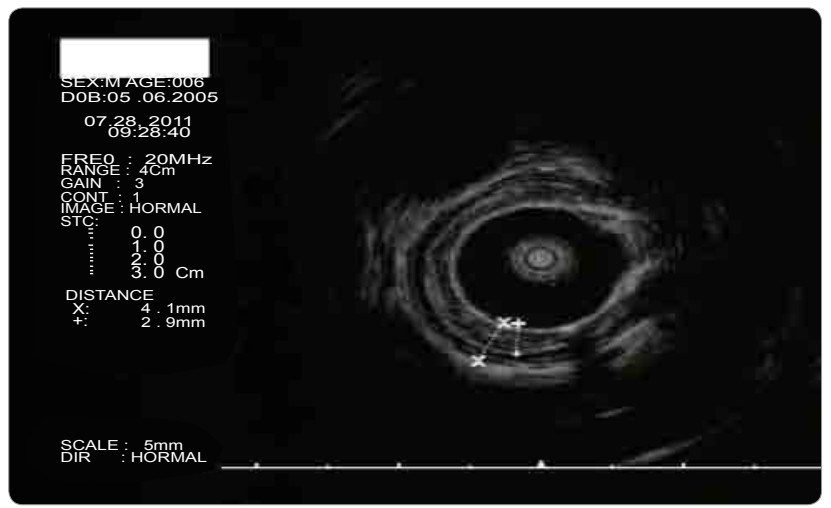

Figure 1b: Endoscopic ultrasound (EUS) exam using $20 \mathrm{MHz}$ high-frequency miniprobe showed thickening of the esophageal wall involving all layers, mucosa, submucosa and muscularis propria.

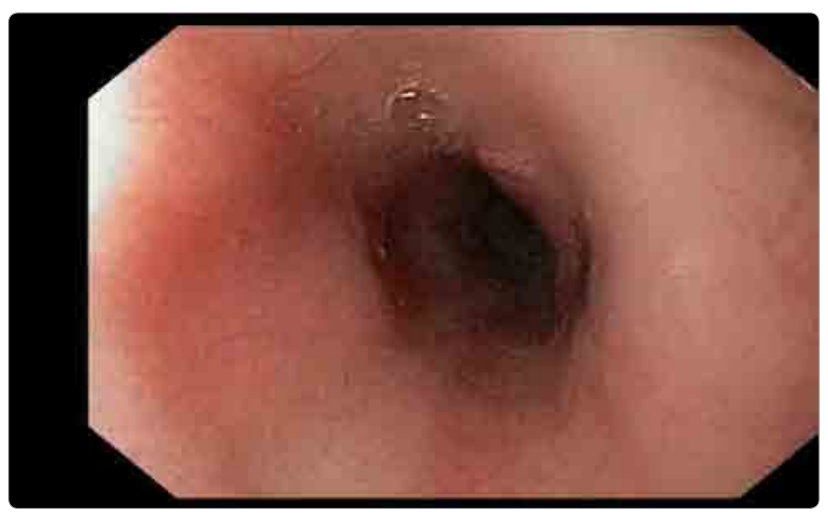

Figure 1c: Follow endoscopy 2 months later, after treatment with oral viscous budesonide, showed healing with resolution of the inflammatory changes.

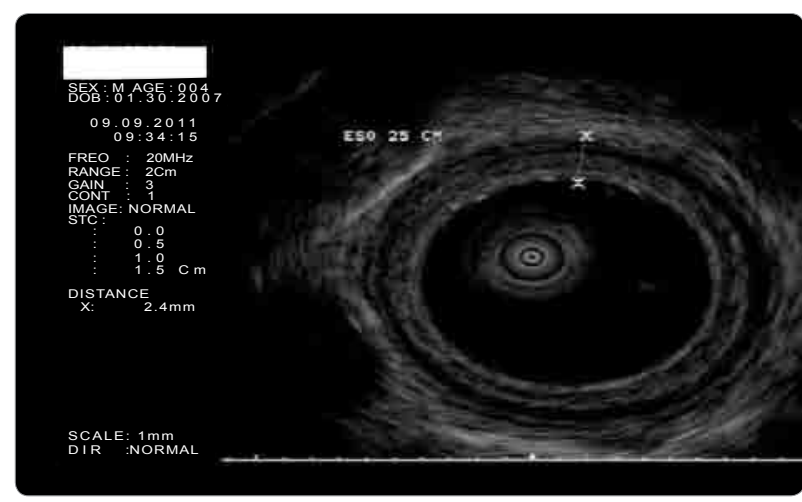

Figure 1d: Reduced thickness of esophageal wall by EUS exam.

the-scope catheter. Changes of the esophageal wall diameter following therapy have shown to correlate well with histologic improvement (Figure 1b, 1d). Meanwhile, a lack of esophageal wall diameter changes is suggestive of an incomplete response to therapy even when mucosal biopsies may be negative for EoE by histologic criteria [8]. Moreover, EUS is useful in differentiating between reflux esophagitis and EoE. Histologic changes in untreated GERD can be difficult to differentiate from EoE. Esophageal wall thickening involving all 3 layers of the esophagus (mucosa, submucosa and muscularis propia) is not typically seen in GERD [9].

\section{Histology}

Again, 15 eosinophils/HPF at the densest area of inflammation remains the minimal threshold for EoE histologic criteria There is no consensus regarding a standardize size to define HPF. This standardized minimal threshold stems from the observation that eosinophilic infiltration caused by GERD typically is in the order of 5 eosinophils/ HPF [1]. Histologic features present in EoE in addition to eosinophilic infiltration include basal cell hyperplasia and elongated connective tissue papillae. Elevated levels of mast cells and lymphocytes are often present. Subepithelial fibrosis, eosinophilic microabscesses and surface layering of eosinophils are features more prominent in EoE in comparison to GERD $[3,10]$ (see Figure 2). In general, data have shown a lack of consistent correlation between degree of inflammation and symptom severity. 


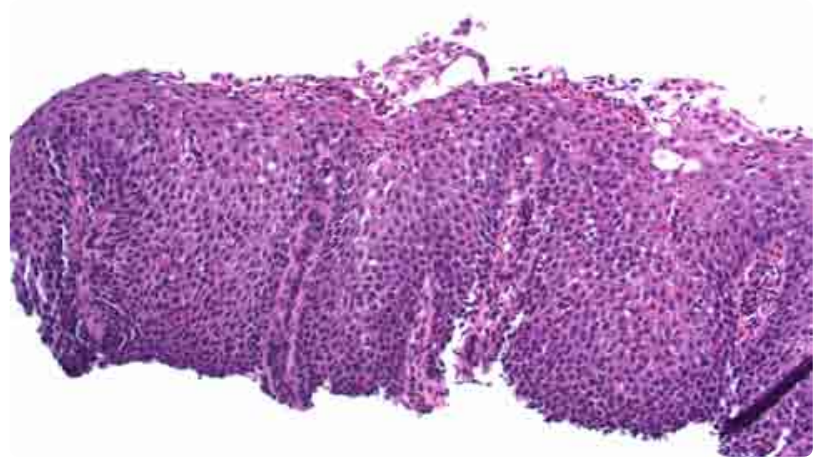

Figure 2: Low magnification exam of biopsy sample from patient identified in Figure 1. Eosinophilic infiltrates in sheets clearly noted with no erosive changes noted. Papillae are elongated and there is some edema noted but these are not salient features on histologic evaluation. These are classic microscopic features of EoE.

\section{Management}

\section{Diet}

Food allergens are the most frequent culprit of inflammation, as the vast majority of pediatric cases resolve with elemental diets [11]. Diet modification-elimination, empiric elimination trials or elemental formulas-is the mainstay of EoE management. Diet elimination involves the collaboration with the pediatric allergist to identify foods most likely driving inflammation. When data from skin testing and patch testing are used, a reported $75 \%$ of cases resolve with food avoidance [3]. Skin and patch testing can be helpful in identifying allergens, although test specificity varies among individual food. The most commonly identified allergen shown to improve avoidance is cow's milk, in $75 \%$ of cases. A major challenge for patients and families is when multiple foods are identified. In a practical sense, multi-food avoidance is highly impractical and makes achieving a nutritionally complete diet a challenge. An alternative approach is a six-food elimination diet. By empirically eliminating the most commonly allergenic foods-cow's milk, soy, nuts, eggs, wheat and seafood-75\% efficacy in symptom reduction and histologic inflammation was reported in one pediatrics study [12]. Again, elemental diets are highly effective in eliminating inflammation. Long-term adherence to such a diet has not been sustainable in most patients. The introduction of an elemental diet, however, is very helpful to confirm whether food allergens are actually playing a role in EoE.

\section{Medications}

Corticosteroids are often used when food allergens are not properly identified, diet modification is not practical for the patient or proves to be insufficient, and when disease is very severe. Swallowed fluticasone spray or budesonide liquid prepared as a slurry acting directly on esophageal mucosa have shown to be effective anti-inflammatory agents with less side-effects of systemic drugs. Fluticasone spray achieved significant improvement in histology and symptomatology in comparison to placebo [13]. A pediatric randomized control trial showed almost $70 \%$ patients had $<6$ eosinophils/HPF following 3 months of budesonide use [14]. One study also suggest that budesonide may beneficially reverse fibrosis [15]. Unfortunately, discontinuation of steroids leads to recurrence of inflammation in the vast majority of patients. Fortunately, the low side-effect profile does allow chronic use as a maintenance medication in some patients with large excretion of drug retrieved in stool. Additionally, absorbed budesonide has the advantage of high first-pass metabolism. The most common side is localized oral or esophageal candidiasis, noted in about $20 \%$ of patients [3]. Overall, long-term studies are limited. Systemic steroids are reserved for severe cases with high grade strictures or severe thickening of the esophagus causing problems such as food impaction.

Medications such as cromolyn and leukotriene receptor antagonists have not shown to be consistently effective in EoE, and are not recommended. Studies of anti-IL-5 therapy are currently underway, and may be beneficial in some patients [1].

\section{Conclusion}

EoE should be considered in any child presenting with upper gastrointestinal symptoms such as dysphagia, emesis and feeding, particularly if there is a history of atopy or reactive airway disease. The prototypical patient will be male, with a personal history of atopy. Most young children will come to attention due to intractable GERD yet the response to antacid therapy is modest at best. Older children will complain of dysphagia, the same complaint is usually noted in adult EoE patients. Upon diagnosis through endoscopy and clinical assessment, identification and avoidance of potential food or environmental triggers are key approaches to management. The role of the allergy specialist is to help isolate specific food and environmental triggers that can be eliminated in an effort to alleviate the child's symptoms. Elemental diets can be used successfully to induce and maintain remission but are difficult to sustain long-term. Swallowed steroids are effective and safe, and can be used as maintenance therapy in some patients. If swallowed steroids are used, care must be used to avoid candidal infections of the mouth and esophagus. EoE is a chronic condition and tends to follow a relapsing and remitting course. Adequate treatment can lead to improved growth and symptom relief and potentially, avoidance of long-term complications in children.

\section{References}

1. Liacouras CA, Furuta GT, Hirano I, Atkins D, Attwood SE, et al. (2011) Eosinophilic esophagitis: updated consensus recommendations for children and adults. J Allergy Clin Immunol 128: 3-20.

2. Noel RJ, Putnam PE, Rothenberg ME (2004) Eosinophilic esophagitis. N Eng J Med 351: 940-941.

3. Putnam PE, Rothenberg ME (2009) Eosinophilic esophagitis: concepts controversies, and evidence. Curr Gastroenterol Rep 11: 220-225.

4. Assa'ad AH, Putnam PE, Collins MH, Akers RM, Jameson SC, et al. (2007) Pediatric patients with eosinophilic esophagitis: an 8-year follow-up. J Allergy Clin Immunol 119: 731-738.

5. Shah A, Kagalwalla AF, Gonsalves N, Melin-Aldana H, Li BU, et al. (2009) Histopathologic variability in children with eosinophilic esophagitis. Am J Gastroenterol 104: 716-721.

6. Schoepfer AM, Gonsalves N, Bussmann C, Conus S, Simon HU, et al. (2010) Esophageal dilation in eosinophilic esophagitis: effectiveness, safety, and impact on the underlying inflammation. Am J Gastroenterol 105: 1062-1070.

7. Subbarao G, Rosenman MB, Ohnuki L, Georgelas A, Davis M, et al. (2011) Exploring Potential Non-Invasive Biomarkers in Eosinophilic Esophagitis: A Longitudinal Study in Children. J Pediatr Gastroenterol Nutr 53: 651-658.

8. Fox VL, Nurko S, Furuta GT (2002) Eosinophilic esophagitis: it's not just kid's stuff. Gastrointest Endosc 56: 260-270.

9. Dalby K, Nielsen RG, Kruse-Andersen S, Fenger C, Durup J, et al. (2010) Gastroesophageal reflux disease and eosinophilic esophagitis in infants and children. A study of esophageal $\mathrm{pH}$, multiple intraluminal impedance and endoscopic ultrasound. Scand J Gastroenterol 45: 1029-1035. 
Citation: Minou Le-Carlson, Antonio Quiros J (2011) Eosinophilic Esophagitis in Children: Updates in Diagnosis and Management. J Aller Ther S8:001. doi:10.4172/2155-6121.S8-001

10. Rothenberg ME (2009) Biology and treatment of eosinophilic esophagitis. Gastroenterology 137: 1238-1249.

11. Markowitz JE, Spergel JM, Ruchelli E, Liacouras CA (2003) Elemental diet is an effective treatment for eosinophilic esophagitis in children and adolescents. Am J Gastroenterol 98: 777-782.

12. Kagalwalla AF, Shah A, Li BU, Sentongo TA, Ritz S, et al. (2011) Identification of specific foods responsible for inflammation in children with eosinophilic esophagitis successfully treated with empiric elimination diet. J Pediatr Gastroenterol Nutr 53: 145-149.
13. Konikoff MR, Noel RJ, Blanchard C, Kirby C, Jameson SC, et al. (2006) A randomized, double-blind, placebo-controlled trial of fluticasone propionate for pediatric eosinophilic esophagitis. Gastroenterology 131: 1381-1391.

14. Dohil R, Newbury R, Fox L, Bastian J, Aceves S (2010) Oral viscous budesonide is effective in children with eosinophilic esophagitis in a randomized, placebocontrolled trial. Gastroenterology 139: 418-429.

15. Aceves SS, Newbury RO, Chen D, Mueller J, Dohil R, et al. (2010) Resolution of remodeling in eosinophilic esophagitis correlates with epithelial response to topical corticosteroids. Allergy 65: 109-116. 